Cuadernos de Gobierno y Administración Pública ISSN: e-2341-4839

https://dx.doi.org/10.5209/cgap.65914

\title{
¿Cómo influye el liderazgo político en el comportamiento electoral subestatal? Una aproximación cualitativa
}

\author{
Miguel Martín de la Cruz'; Jesús García de Madariaga²
}

Recibido: 14/03/2019 / Aceptado: 24/06/2019

Resumen. Cuando los electores deciden su voto en unas elecciones municipales ¿lo hacen pensando en el candidato o en el partido? ¿O bien hay alguna otra variable crítica que entra en juego al decidir el voto en el ámbito local? En esta investigación realizamos una aproximación cualitativa al fenómeno del voto subestatal. Si bien la evaluación del candidato es un factor particularmente importante a la hora de decidir el voto en unas elecciones municipales, éste no es el único y no siempre es el principal elemento decisorio. La revisión del marco teórico existente sobre el comportamiento electoral y las variables / dimensiones que operan en el proceso decisional en elecciones municipales es muy escaso. Por ello planteamos un estudio de casos mediante la celebración de grupos de discusión en tres municipios con diferentes realidades históricas respecto al liderazgo personal de los candidatos.

Palabras clave: Liderazgo; elecciones municipales; elecciones de segundo orden; candidatos; personalización y España.

\section{[en] How does political leadership influence sub-state electoral behavior? A qualitative approach}

\begin{abstract}
When the voters decide their vote in council elections, do they thinking about the candidate or the party? Or is there any other critical variable that comes into play when deciding the vote at the local level? In this research we perform a qualitative approach to the phenomenon of sub-state voting. Although the evaluation of the candidate is a particularly important factor when deciding the vote in a council elections, this is not the only one and is not always the main decision element. The review of the existing theoretical framework regarding electoral behavior and the variables / dimensions that operate in the decision-making process in council elections are very limited. Therefore, we propose a case study by holding focus groups in three municipalities with different historical realities regarding the personal leadership of the candidates.
\end{abstract}

Keywords: Leadership; local elections; second-order elections; candidates; personalization and Spain.

Sumario: 1. Introducción. 2. Los candidatos en las elecciones municipales. 3. Las dimensiones de la personalidad en los candidatos municipales. 4. Preguntas de la investigación. 5. Metodología de la investigación. 6. Resultados. 7. Conclusiones. 8. Bibliografía.

\footnotetext{
$1 \quad$ Universidad Complutense de Madrid miguelmartin@ccee.ucm.es

2 Miembro del Instituto Complutense de Ciencia de la Administración Universidad Complutense de Madrid jesgarci@ucm.es
} 
Cómo citar: Martín de la Cruz, M.; García de Madariaga, J., ¿Cómo influye el liderazgo político en el comportamiento electoral subestatal? Una aproximación cualitativa, en Cuadernos de Gobierno y Administración Pública 6-2, 163-184.

\section{Introducción}

En este trabajo se investigan las variables que intervienen en el proceso decisional del voto en unas elecciones municipales, la relevancia del candidato en dicho proceso y el conjunto de dimensiones que la configuran.

El papel de los candidatos en la decisión de voto es una pregunta clásica en los estudios electorales. No son pocos los autores que han destacado la importancia del candidato a la hora de determinar quién gana y quién pierde las elecciones (King, 2002; Rico, 2009); e incluso ya se han realizado estudios empíricos que vienen a demostrar la influencia del liderazgo personal en las elecciones municipales, asumiendo que dicho liderazgo está correlacionado directamente con el tamaño del municipio (Riera, 2017).

En este texto se profundiza -en términos cualitativos- en la variable liderazgo personal, su importancia dentro del discurso de los electores y su composición, yendo más allá del factor "cercanía" como único elemento del liderazgo. Algo que, si bien está presente en todo momento y es nuclear en la definición de la variable, no puede definir por sí solo al liderazgo personal del candidato.

Las elecciones municipales no han sido hasta hace unos años un área de investigación de especial interés para los académicos de la ciencia política. Tal como se ha afirmado, en los sistemas democráticos los comicios locales permanecen en su infancia (Marschall, Sha y Runil, 2011). En España la situación no es mucho mejor. Los estudios realizados al respecto se centran en una sola elección y cuatro ciudades (Barberá, 2010) o en una comunidad autónoma (Martínez Fuentes y Ortega, 2010), o trabajan con datos agregados (Delgado, 1997; 2010). Y en todos los casos son estudios de carácter cuantitativo. No he encontrado trabajos académicos cualitativos que realicen una aproximación a las variables presentes en el proceso de decisión del voto en unas elecciones municipales.

El resto del artículo se estructura en ocho epígrafes: Los dos siguientes describen el marco teórico existente sobre la investigación y las preguntas de investigación. A continuación, se plantean las cuestiones a las que ha pretendido dar respuesta esta investigación y se expone la metodología utilizada. El epígrafe 6 se dedica a la exposición de los principales resultados alcanzados; y el último detalla las conclusiones a las que se ha llegado y se establecen las líneas de actuación futuras.

\section{Los candidatos en las elecciones municipales}

De manera general las publicaciones sobre la influencia de los candidatos sobre el voto han tratado de ofrecer una respuesta de tipo normativo a la pregunta de cómo los procesos electorales determinan quién debe llevar a cabo las diferentes actuaciones políticas. La respuesta a dicha pregunta está condicionada a su vez por la relevancia que se otorgue a los líderes políticos en los procesos electorales. En este sentido existen dos corrientes de estudio. La primera, la de las elecciones de segundo orden (Reif 
y Schmitt, 1980), aplicable normalmente a los comicios europeos, pero también a los locales, carece de interés para este estudio. La segunda, centrada en la personalización, en parte coincidente con la teoría de la presidencialización de las elecciones en países como Estados Unidos, es en la que fundamenta esta investigación.

La personalización o presidencialización de la política consiste en el estudio de la importancia que logran los candidatos o líderes en la acción de gobierno, en la pugna electoral e incluso en la dirección de sus partidos (Muchan 1993; Hazan 1996; Costa Lobo, 2014). Guillem Rico (2009: 13 y 23) ha definido la personalización como "el creciente impacto de los líderes en las decisiones de los votantes en relación al peso de otros factores, y en particular el de las identidades partidistas y otras predisposiciones políticas", incluyendo en "la manera en que los ciudadanos procesan la información política, la forma en que ven a los partidos, juzgan a los gobiernos y se forman opiniones sobre los temas de debate político".

En un principio, la estructura de gobierno se consideraba importante para explicar el fenómeno de la personalización en la medida que se atribuía casi exclusivamente a los sistemas presidencialistas como el americano (Bartle y Crewe, 2002). Sin embargo, la monopolización de este planteamiento por parte de estos sistemas de gobierno ha dejado de tener validez para extenderse a los de carácter parlamentario (Costa Lobo y Curtice, 2014). Incluso numerosas investigaciones han estudiado el impacto de los candidatos en las elecciones legislativas (Graetz y McAllister, 1987; Bean, 1993; Clarke, 2000).

El caso español no es una excepción en la transversalización de la teoría de la personalización a sistemas parlamentarios en la política. Desde el comienzo de nuestra nueva etapa democrática, los líderes políticos disfrutan de elevados niveles de poder e influencia; tienen autonomía casi completa a la hora de conformar su equipo de campaña electoral y controlan férreamente la organización de su partido político. Así se ha podido constatar desde el comienzo de la democracia actual con figuras como Felipe González, José María Aznar, o José Luis Rodríguez Zapatero, capaces de atraer al voto indeciso y abstencionista en momentos claves de sus respectivas formaciones políticas (Rico, 2007).

Buena parte de la literatura disponible sobre la personalización del voto en las Elecciones en España se centra en contextos de tipo nacional o autonómico. Pero si en algún nivel se puede considerar que se produce una estrecha relación entre el comportamiento electoral y la teoría de la personalización / presidencialización es en el ámbito local o de elecciones municipales. Esta relación se produce por varios motivos:

- La relevancia de la figura del alcalde en los procesos locales. Como indicaba Jordi Capo (1991: 158) "(los alcaldes) son fuertes, son estables, tienen recursos y capital político”.

- La propia configuración de las elecciones locales, en clave presidencialista, otorgando amplios poderes al alcalde (Vanaclocha, 1997).

- Solo los cabezas de lista pueden competir por la alcaldía. Aunque acuden en una plancha electoral con el resto de compañeros, es el número uno quien accede a la alcaldía.

A pesar de la configuración parlamentaria de los comicios municipales establecida en la Ley Electoral vigente, la competición electoral se ha desarrollado hasta 
ahora en clave presidencialista entre los diferentes candidatos identificados con sus respectivos partidos (Márquez, 2007). Mientras que el resto de la lista electoral aspira a formar parte del equipo de gobierno a través de una concejalía. Por ello, los partidos tratan de seleccionar candidatos con elevado conocimiento en su localidad, especialmente cuando el propio partido carece de implantación local (Martínez Fuentes, 2008). Es decir, la campaña electoral de ámbito municipal gira en torno al candidato y en la mayoría de las ocasiones éste disfruta de una elevada autonomía para dirigirla. Este fenómeno se traduce en dos factores (Martínez Fuentes, 2010):

- La tendencia a resaltar las cualidades y trayectoria política, personal y social del candidato, mientras el resto de miembros de la lista son relegados a un segundo plano.

- La autonomía de los candidatos municipales para "personalizar" su programa y discurso, enriqueciendo las consignas provenientes de niveles nacionales y/o autonómicos del partido donde milita (aun con el riesgo de, en ocasiones ir "en contra" de las líneas de comunicación del partido a nivel estatal). Como se muestra más adelante, esta situación es más probable cuando el candidato supera ampliamente a la marca en la construcción mental del elector.

Esta personalización confiere a los candidatos la posibilidad de establecer relaciones de poder dentro de la organización de los partidos que puede ir más allá del propio municipio, hasta el punto de que hay alcaldes y candidatos que se convierten en figuras políticas nacionales (Martínez Fuentes y Ortega, 2010). En España podemos acudir a ejemplos como el de Ada Colau, Manuela Carmena o Esperanza Aguirre.

Por otro lado, la cercanía de los candidatos con los electores les confiere una accesibilidad que contrasta con la distancia inevitable de quienes compiten en elecciones de ámbito nacional (Barberá, 2010). La cercanía unida al recurso del trato directo que pueden realizar los candidatos municipales en un contexto de competición centrado en los líderes de los partidos a nivel local, convierte a éstos en elementos fundamentales para la decisión del voto (Freire, 2005). Por lo tanto, las elecciones locales enfatizan el trato directo de los candidatos con el electorado, lo que incrementa -en principio-su relevancia entre los factores de voto, en detrimento de otros elementos como puede ser la marca / partido, el entorno social y/o económico, entre otros.

\section{Las dimensiones de la personalidad en los candidatos}

El estudio en España del peso que ejerce la valoración de los candidatos municipales en comparación con el resto de determinantes del voto ha sido bastante escaso durante años. Esta carencia ha tratado de cubrirse mediante argumentos especulativos o mediante su restricción a un ámbito territorial concreto, o con la extrapolación a nivel local de la valoración de los líderes nacionales de los partidos más relevantes (Martínez Fuentes y Ortega 2010). Los resultados no resultan satisfactorios por la singularidad del voto local y la necesidad de profundizar en un escenario político municipal que no es estable ni territorial ni temporalmente. En los estudios de ámbito municipal más recientes sí se verifica la hipótesis de la importancia de la valora- 
ción de los candidatos en la probabilidad de votar a un determinado partido. Así mismo se verificó que "la magnitud de la personalización del voto en las elecciones municipales en España se ve modificada por el tamaño del municipio y el paso del tiempo" (Riera, 2017: 11).

Así mismo en el estudio de Martínez Fuentes y Ortega (2010) se pudo contrastar que el efecto del candidato sobre el comportamiento electoral se mantiene incluso cuando incorporamos otros factores sociodemográficos del votante, tales como el sexo, la situación laboral, la educación o la edad del elector; e incluso por factores de tipo político, como su ideología. El planteamiento de esta investigación trabaja con los diferentes estudios disponibles en el banco de datos del CIS, lo que impide indagar si existen otras variables o dimensiones relevantes en el proceso de decisión del voto a nivel municipal; incluso yendo más allá, la propia definición de la personalización del voto queda acotada al tamaño del municipio y la valoración del candidato (habitualmente el CIS trabaja con escalas de 0 a 10). Esto no hace sino reforzar las afirmaciones realizadas respecto a las dificultades inherentes al estudio de elecciones municipales, por la falta de investigaciones específicas y particularizadas para elecciones de segundo orden y más concretamente sobre la teoría de la personalización en este tipo de comicios.

El estudio de las dimensiones de la personalidad en el caso de elecciones de primer orden sí ha sido objeto de atención por parte de los investigadores -aunque menor que otras áreas de estudio político electoral- y que quedan recogidas en la siguiente Tabla 1.

Tabla 1. Investigaciones sobre las dimensiones de la personalidad en las elecciones de primer orden.

\begin{tabular}{|c|c|c|c|c|c|c|c|}
\hline & Competencia & $\begin{array}{c}\text { Integridad / } \\
\text { Honradez }\end{array}$ & \begin{tabular}{|c|} 
Liderazgo / \\
Carisma
\end{tabular} & Empatía & $\begin{array}{c}\text { Apariencia } \\
\text { personal }\end{array}$ & Responsabilidad & Otras \\
\hline Miller\&Miller (1976) & $\checkmark$ & & $\checkmark$ & & $\checkmark$ & $\checkmark$ & $\checkmark$ \\
\hline Nimmo\&Savage (1976) & & $\checkmark$ & $\checkmark$ & $\checkmark$ & & & \\
\hline $\begin{array}{l}\text { Kinder, Abelson } \\
\text { y Fiske (1979) }\end{array}$ & $\checkmark$ & $\checkmark$ & & & & & \\
\hline Markus (1982) & $\checkmark$ & $\checkmark$ & & & & & \\
\hline Kinder (1986) & $\checkmark$ & $\checkmark$ & $\checkmark$ & $\sqrt{ }$ & & & \\
\hline $\begin{array}{l}\text { Miller, Wattenberg y } \\
\text { Malenchuk (1986) }\end{array}$ & $\checkmark$ & $\checkmark$ & $\checkmark$ & & $\checkmark$ & $\checkmark$ & \\
\hline $\begin{array}{l}\text { Lodge, McGraw y Stroh } \\
\text { (1989) }\end{array}$ & $\checkmark$ & $\checkmark$ & & & & & \\
\hline McCann (1990) & $\checkmark$ & $\checkmark$ & $\checkmark$ & $\checkmark$ & & & \\
\hline $\begin{array}{l}\text { Caprara, Barbaranelli y } \\
\text { Zimbardo }(1997,2002)\end{array}$ & & & & & & & $\checkmark$ \\
\hline Funk (1999) & $\checkmark$ & $\checkmark$ & $\checkmark$ & $\checkmark$ & & & \\
\hline $\begin{array}{l}\text { Pancer, Brown y Widdis } \\
\text { Barr (1999) }\end{array}$ & $\sqrt{ }$ & $\checkmark$ & $\checkmark$ & & & & \\
\hline
\end{tabular}




\begin{tabular}{|l|l|l|l|l|l|l|l|}
\cline { 2 - 7 } \multicolumn{1}{c|}{} & Competencia & $\begin{array}{c}\text { Integridad / } \\
\text { Honradez }\end{array}$ & $\begin{array}{c}\text { Liderazgo / } \\
\text { Carisma }\end{array}$ & Empatía & $\begin{array}{c}\text { Apariencia } \\
\text { personal }\end{array}$ & Responsabilidad & Otras \\
\hline $\begin{array}{l}\text { Brettschneider\&Gabriel } \\
(2002)\end{array}$ & $\checkmark$ & $\checkmark$ & $\checkmark$ & & $\checkmark$ & & \\
\hline Newman (2003) & $\checkmark$ & $\checkmark$ & & & & & \\
\hline $\begin{array}{l}\text { Ohr \& Oscarsson } \\
(2003)\end{array}$ & $\checkmark$ & $\checkmark$ & $\checkmark$ & $\checkmark$ & & & $\checkmark$ \\
\hline $\begin{array}{l}\text { Clarke, Sanders, } \\
\text { Stewart y Whiteley } \\
(2004)\end{array}$ & $\checkmark$ & & & & & & \\
\hline
\end{tabular}

Fuente: Elaboración propia.

La sistematización de los elementos recogidos en la tabla 1 en la que se resumen las quince teorías reseñadas, viene a dar la siguiente jerarquización:

- Dos de estos elementos aparecen señalados de forma muy destacada sobre el resto.

- La competencia es la dimensión que se sitúa en primer lugar con 13 menciones sobre 15.

- En segundo lugar, se sitúa la integridad/honradez con 12 menciones.

- Otros cuatro aparecen señalados por varias de las teorías, aunque a bastante distancia de los anteriores.

- El liderazgo/carisma, con 8 menciones.

- La empatía, con 4.

- La apariencia personal y las características socio demográficas, con 2.

- La responsabilidad, con 2.

- Otros varios aparecen señalados de forma particular por alguna de dichas teorías: la confianza, la receptividad, el atractivo personal

Tal como se indicaba en los párrafos anteriores, no existen -o al menos no con la suficiente relevancia- estudios sobre las dimensiones de la personalidad de los candidatos en elecciones subestatales, concretamente para el ámbito municipal. De ahí la relevancia de la presente investigación, que pretende arrojar algo más de luz sobre un área escasamente estudiada pero de enorme interés para las formaciones políticas cuando eligen y promocionan a sus candidatos en los diferentes municipios.

\section{Preguntas de investigación}

Como ya se indicó en el epígrafe anterior, ha quedado patente que la valoración de los candidatos locales tiene una influencia significativa en el voto en las elecciones municipales. Esta influencia permanece al incorporar en el análisis de va- 
riables sociodemográficas de los votantes e incluso su ideología. Además, se verifica que el efecto de la personalización tiende a reducirse conforme aumenta el tamaño del municipio y mayor importancia se otorga al voto ideológico (Riera, 2017).

Esta investigación se centra en un diseño exploratorio sobre dos cuestiones. En primer lugar, trata de profundizar en el discurso social que se produce al hablar del voto municipal y del proceso de decisión del mismo. Ya ha quedado demostrado que el binomio marca - candidato tiene una elevada relevancia (Rico, 2010; Costa Lobo, 2014), pero ¿son las únicas variables que influyen en el voto municipal? En segundo lugar, busca ahondar en la identificación de las variables que intervienen en la personalización del voto municipal, toda vez que los estudios empíricos realizados sobre la personalización se han basado en elecciones de primer orden (Crewe, 2002; Costa Lobo ,2014); mientras los de ámbito municipal han confirmado sus hipótesis a partir de estudios empíricos y fuentes secundarias de información de naturaleza eminentemente cuantitativa (Riera, 2017). En definitiva, ¿qué variables configuran la dimensión de la personalización del voto municipal?

\section{Metodología de la investigación}

Durante las últimas décadas las investigaciones realizadas con relación a la personalización en las elecciones de primer nivel -e incluso en las de segundo nivel- se han apoyado en una metodología cuantitativa, bien mediante el tratamiento de bases de datos obtenidas de instituciones u organismos públicos, bien a través de diseños concluyentes mediante encuestas.

La investigación llevada a cabo es - por el contrario- de naturaleza exploratoria y carácter cualitativo, en la que se pretende realizar una aproximación y profundización en la personalización del voto como variable nuclear y su definición a partir de un conjunto de dimensiones. Todo ello centrado en el ámbito municipal y en las elecciones locales.

El marco de la investigación cualitativa se diseñó siendo conscientes de la necesidad de contar con representación de los diferentes discursos relativos a las elecciones locales y a las variables que intervienen en el proceso decisional del voto.

A partir de la revisión de las diferentes teorías sobre la personalización del voto y la constatación de la ausencia de un marco teórico en el ámbito de las elecciones municipales, se optó por el estudio de casos que posibilitasen la generación de teorías al respecto.

El estudio de casos se revela como una herramienta de gran valor para nuestra investigación, toda vez que a través del mismo es posible registrar la conducta de los individuos -y profundizar en la misma- mientras que los métodos cuantitativos se centran en la información obtenida de las encuestas mediante cuestionarios estructurados (Yin, 1989). Además de ser considerado apropiado para investigaciones exploratorias (Chetty, 1996), también ha mostrado su capacidad para la generación de teorías (Gersick, 1988). Además, según Glaser y Stratuss (1967: 30), la investigación que pretende contribuir a generar teorías que alcancen un cierto grado de aplicabilidad general, pueden basarse en un número limitado de casos, ya que "un solo caso puede indicar una categoría o propiedad conceptual y unos cuantos casos más pueden confirmar esa indicación". 
La generalización a partir del estudio de casos no consiste en una "generalización estadística" como en las encuestas, sino que es una "generalización analítica" (Yin, 1998). De esta forma, los resultados del estudio de un caso (o casos) pueden generalizarse a otros que presentan condiciones teóricas similares.

Se planificó la celebración de nueve grupos de discusión distribuidos a partes iguales en tres puntos de muestreo que sirviesen como estudio del caso de las diferentes realidades municipales y su hipotética relación con la personalización del voto:

- Las Palmas de Gran Canaria, por ser un municipio de tamaño grande (más de 200.000 electores) en el que se han producido a lo largo de los años etapas con -aparentemente- un liderazgo personal en sus representantes municipales; alternando con etapas con ausencia de dicho liderazgo.

- Santa Lucía de Tirajana, como municipio de tamaño intermedio $(<100.000$ electores) con una trayectoria ideológica histórica que se ha combinado con liderazgos personales fuertes.

- Por último, Teguise, municipio de la Isla de Lanzarote en el que desde hace más de 30 años se produce un relevo en la alcaldía por candidatos con un fuerte componente de liderazgo personal. Además, representa adecuadamente la figura del municipio de pequeñas dimensiones ( $<15.000$ electores).

En cada municipio se llevaron a cabo tres grupos de discusión segmentados por las edades de los participantes:

- Un grupo formado por electores de 18 a 30 años.

- Otro grupo integrado por personas de 31 a 49 años.

- Y un tercer grupo con votantes de 50 o más años.

Todos los grupos celebrados tenían un conjunto de características compartidas:

- Representación equiparada por sexos (50/50).

- En cada segmento de edad se garantizó la presencia de los diferentes roles situacionales: estudiantes, parados, con empleo por cuenta ajena, por cuenta propia, responsables del hogar, jubilados, etc.

- Cada grupo tuvo una representación de simpatizantes de los principales partidos presentes en el municipio.

- Todos los participantes debían ser residentes habituales en su municipio y tener derecho a voto en las elecciones municipales.

- Así mismo, con el objeto de lograr una cobertura geográfica por entidades y barriadas, cada grupo contaba con la presencia de residentes en los principales barrios y entidades de cada municipio.

La selección y captación de los asistentes a los grupos fue realizada mediante un sistema de reclutamiento basado en un breve cuestionario donde se cualificaba al individuo. Si cumplía con las condiciones buscadas se le invitaba a asistir a un grupo en concreto. A aquellos que aceptaron se le citó en día, hora y lugar determinado y se registraron sus datos de contacto. Todo el proceso de selección se realizó en la vía pública del municipio en cuestión, mayoritariamente en zonas comerciales con un elevado tránsito viario. 
La celebración de los grupos se llevó a cabo -en los meses de junio y julio de 2018- en salas especialmente acondicionadas al efecto, teniendo una duración media de 120 minutos por grupo. Las más de 18 horas de grabación de audio generadas fueron transcritas, conformando la fuente de análisis principal.

\section{Resultados}

\subsection{La personalización del voto en la ecuación electoral}

En todos los grupos realizados, cuando se plantea el sistema de decisión del voto, nos encontramos con una opinión que alcanza un respaldo significativo entre quienes participan de los mismos: "En las elecciones locales se vota a la persona."

El que dicha opinión no fuera rebatida por quienes se decantaron por opiniones diferentes en el transcurso del intercambio dinámico de opiniones indica un reconocimiento implícito de dos sentidos que se contienen en esta proposición y que alcanzan un reconocimiento compartido por todos:

- Se percibe que las elecciones municipales marcan significativas diferencias con respecto a las de ámbito nacional o autonómico. Algo que ya apuntaban los estudios de Jordi Capo (1991) o Vanaclocha (1997) al referirse a la figura del alcalde. Algunas significativas particularidades que tienen que ver con los asuntos locales y con la percepción de mejora en el cotidiano de los barrios (desde las infraestructuras a la vida social) o en los desplazamientos por la ciudad (desde el transporte al entretenimiento). Estas diferencias vienen respaldadas estadísticamente cuando se analiza la distinta configuración del voto a nivel local, regional y nacional en los tres municipios.

Gráfico 1: Resultados electorales en los comicios municipales y estatales de 2011 y 2015.

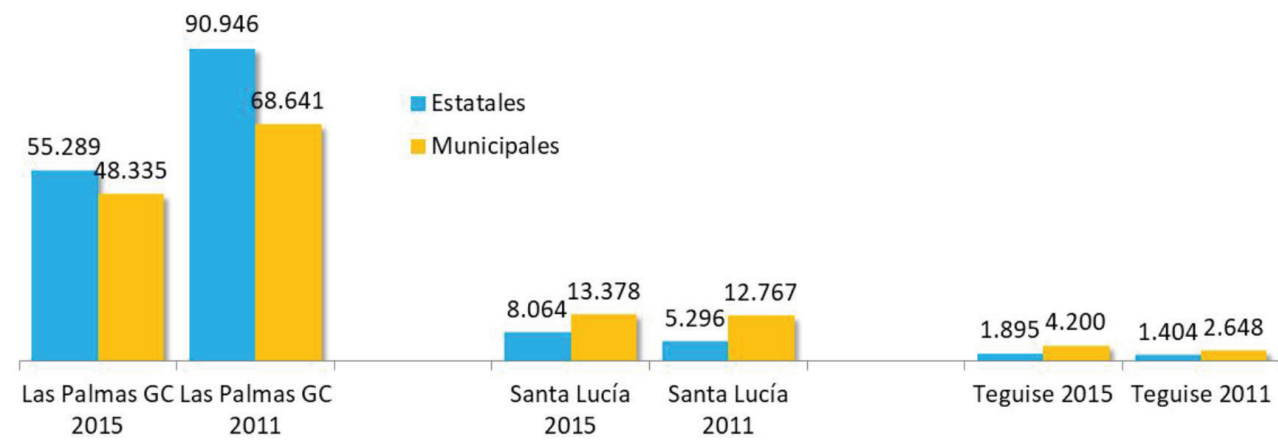

Fuente: Ministerio del interior. Procesos Electorales. Resultados Electorales. Elaboración propia.

- Y se señala como principal diferencia la importancia que se le atribuye a la "condición personal" del candidato o candidata, en este tipo de comicios electorales. Una importancia que se correlaciona con el mayor nivel de conocimiento y de cercanía que se aprecia con ellos. Algo que justifica una mayor 
atribución de peso a la valoración personal con respecto a los mismos y que ya enunciaba Freire al referirse a los factores de voto (2005).

Cuando se avanza en el intercambio dinámico de opiniones dentro de los grupos dicha importancia queda relativizada al abrirse la matriz de sentido de la configuración del voto a otras variables nucleares. Así se comprueba en el discurso de nuestros informantes en los tres casos de los municipios consultados.

En cada uno de ellos las dinámicas de grupo se orientaron a propiciar un discurso flotante sobre el sistema de decisión del voto a nivel local de tal manera que la configuración del mismo quedara abierta a su conformación espontánea desde las variables que vienen a definir la percepción del sistema de decisión a nivel de cada municipio. El análisis del discurso generado en torno a la evolución percibida viene a redundar en que el diagnóstico de dicha evolución se sustenta en el siguiente conjunto de variables:

- La marca (el partido)

- El liderazgo (la persona candidata)

- La expectativa de cambio ante una situación que se considera perjudicial o inmantenible (expresión utilizada en los grupos de discusión celebrados de manera espontánea).

- La conformidad con la opción tomada por las mejoras experimentadas.

La que se decanta como prioritaria es la marca. Ésta aparece como el principal recurso:

- del que valerse para decantar la decisión de voto cuando en la elección no inciden notablemente otros factores intervinientes decisivos.

- en el que mantenerse cuando se valora positivamente el resultado de la legislatura.

- al que volver cuando se han frustrado las expectativas de cambio

"Las Palmas se ha demostrado como un electorado propenso a votar al PP".

"Las Palmas ha venido siendo mayoritariamente del PP".

"Es algo que se ha venido palpando en la calle y que se ha visto reflejado en lo sucedido en las elecciones y en los gobiernos que hemos tenido...De hecho, hemos estado gobernados por el PP la mayoría de todos estos años”.

"Aquí (Santa Lucía) se ha votado a Nueva Canarias desde hace muchos años, casi desde el principio (de los Ayuntamientos democráticos). Parece que la mayoría de la gente estuviese adscrita a esta marca”.

El mayor peso de la marca hegemónica sobre el liderazgo viene a quedar comprobado en dos momentos electorales concretos en el caso de Las Palmas de Gran Canaria:

- El PP mantiene la mayoría absoluta en el Ayuntamiento cuando José Manuel Soria es sustituido como candidato a la alcaldía por Josefa Luzardo; una política joven con una marcada carencia de liderazgo. 
- El PP recupera la mayoría absoluta tras la frustración experimentada con Jerónimo Saavedra, a pesar del bajo nivel de reconocimiento y el escaso nivel de liderazgo atribuido al candidato presentado por el partido.

"A Pepa Luzardo se la votó porque suponía la continuidad con el gobierno del PP protagonizado por José Manuel Soria durante varias legislaturas".

"A Cardona no se le votó a él mismo. Ganó la alcaldía porque se votó al PP".

La marca ya fue considerada como la variable principal en el proceso decisional por la teoría partidista de la Escuela de Michigan (Campbell, 1960), aunque Roche (2008) afirma que el voto es considerado como un mecanismo de afirmación a una clase social que se refleja en el voto a una marca / partido concreto.

La variable que destaca en segundo lugar es el liderazgo.

La capacidad de liderazgo y el carisma personal atribuido a Jerónimo Saavedra intervino decisivamente en su elección como alcalde de la ciudad en 2007.

"En el caso de Saavedra sí que se votó a la persona. Era alguien que gozaba de un gran reconocimiento como persona y como político".

"Le votó incluso mucha gente que había venido votando habitualmente al PP... Eso fue lo que le permitió obtener la mayoría absoluta en una ciudad que había demostrado una clara tendencia a votar al PP...Algo que había sucedido en otros sitios de la Península como por ejemplo en Córdoba con Julio Anguita".

El hecho de alcanzar mayorías absolutas en ciudades caracterizadas por la tendencia a votar a marcas políticas con sentido opuesto aparece como una clara corroboración de la fuerza que puede llegar a alcanzar el liderazgo en las elecciones municipales. Algo que se verifica en Teguise, con una tendencia de voto "estatalista" en elecciones generales.

"En las generales yo voto al PP, pero aquí (municipales) votó a Oswaldo (Betancort)" (Teguise).

"En el caso de Saavedra sí que se votó a la persona. Era alguien que gozaba de un gran reconocimiento como persona y como político".

La afirmación sobre las cualidades del candidato en unas elecciones municipales que refleja Martínez Fuentes (2010) y su capacidad para personalizar el voto tienen su reflejo en la fuerza con que emergió el liderazgo político de José Manuel Soria como alcalde de Las Palmas de Gran Canaria durante sus dos primeras legislaturas reafirmó la opción del voto hacia el PP en la ciudad, reforzando sus mayorías absolutas consecutivas hasta el punto de que la opinión mayoritaria es que este partido no hubiera perdido la alcaldía si José Manuel Soria se hubiera mantenido como candidato a la misma.

“A José Manuel Soria se le votó por la orientación del voto hacia el PP después de que las otras alternativas no consiguieran hacer ver los resultados en la mejora de la ciudad por falta de tiempo para su materialización. Pero una vez que él reafirmara su liderazgo político en las primeras legislaturas, la gente le votaba mayoritariamente a él, incluso personas que se sentían más afines a otras ideologías". 
"Su liderazgo alcanzó tanto ascendente que en algún momento se hablaba de él en un sentido enfático como de "el alcalde de Las Palmas", y no sólo en la ciudad sino también en la Isla e incluso en la provincia".

"De hecho, el PP hubiera mantenido la alcaldía de la ciudad si él se hubiera mantenido como candidato".

El liderazgo político atribuido a Antonio Morales entre votantes de izquierdas y/o nacionalistas en el conjunto de Gran Canaria por los resultados obtenidos en Agüimes, el municipio del que había sido alcalde durante muchas legislaturas, se argumenta por nuestros informantes como explicación al significativo incremento del voto a Nueva Canarias, el partido al que pertenece, sobre todo a nivel insular, pero también en la capital.

"Yo antes votaba a CC, pero en las últimas voté a Nueva Canarias. El Presidente del Cabildo es un buen presidente. Antonio Morales".

"Yo voté Nueva Canaria por la persona, por Morales, he visto lo que hizo".

"Yo también. Y voté a Antonio porque de buena mano ya sabía quién era, porque tengo muchos amigos en Agüimes y allí ha hecho muchas cosas".

En tercer lugar, se sitúa la expectativa de cambio, a la que se atribuye un papel decisivo en tres momentos de la evolución experimentada:

- Propició la llegada de Jerónimo Saavedra a la alcaldía de la ciudad ante la decepción provocada por el relevo de José Manuel Soria en el PP con Pepa Luzardo.

"Se había votado a Pepa Luzardo porque se la percibía como la continuidad del gobierno mantenido por José Manuel durante varias legislaturas. Pero con el paso de los años esa percepción de continuidad se fue perdiendo y ella fue incapaz de aguantar el tipo por falta de carisma y de capacidad de gestión. Su permanencia se hizo inmantenible incluso para votantes de siempre del PP. Se había extendido la expectativa de cambio, el PP no supo responder a esa expectativa y el PSOE acertó presentando a Jerónimo Saavedra".

- Dio paso a probar otras alternativas votando a nuevos partidos, en las últimas elecciones municipales, ante la pérdida de apoyos de los partidos de siempre. Ello provocó la pérdida de la capacidad de gobierno por parte del PP, al transferirse parte sus votos habituales a un partido de reciente formación en el espectro de la derecha insular, Unidos por Gran Canaria, y propició la vuelta del PSOE en minoría al gobierno municipal con el apoyo de dos fuerzas políticas emergentes: Las Palmas Puede y Nueva Canarias.

- Por último, la vuelta a las marcas a las que se ha venido votando habitualmente tras la frustración de las expectativas de cambio aparece señalada de forma significativa entre nuestros informantes.

"Yo, desde mi punto de vista, viendo esto, creo que es normal que te posiciones en un partido político. Estudiarás tus opciones, pero serán en un partido... Se va a reagrupar el voto". 
"Esto es una ola. Hay mucha gente que no sabe y entonces dice: más vale lo malo conocido que lo bueno por conocer".

"No ha habido suerte en el cambio; ha sido malo. Entonces yo creo que volvemos a lo anterior".

"Mucha gente volverá al partido que votó en su momento".

Como alternativa a ello se señala a la aparición de nuevos liderazgos políticos.

"Y entonces ¿qué hacemos: volver a lo mismo? "Si no aparecen caras nuevas, puede ser".

"Es que tenemos un problema bastante gordo con eso y es que realmente no tenemos cambio. Vuelven a estar los mismos de siempre".

"Es muy decepcionante cuando hay elecciones, y no has quedado contento con lo que votaste, el no saber a quién votar porque realmente no conozco a nadie que me convenza. Si lo conozco lo voto; si no lo conozco, voto por el partido que he votado normalmente".

"Te refugias en la marca de siempre porque no conoces nadie".

"Si hay alguien que me convence doy el paso; si no, vuelvo a donde estaba. Más vale lo malo conocido que lo bueno por conocer".

En último lugar, y como variable concomitante, aparece la conformidad con la opción tomada.

En el caso de Las Palmas de Gran canaria este factor aparece sólo como una variable concomitante a las de la marca y el liderazgo. De hecho, sólo alcanza un peso decisivo para explicar la evolución experimentada en la configuración del voto local en este municipio cuando se consolida la opción electoral por el PP tras la consolidación del liderazgo de José Manuel Soria como "el alcalde de Las Palmas". Las sucesivas legislaturas en que se mantiene como alcalde de la ciudad van reforzando la opción electoral tomada por la mayoría de la población.

La conformidad con la opción tomada, sin embargo, coprotagoniza, junto con la marca, la evolución experimentada en la configuración del voto local en Santa Lucía de Tirajana.

Profundizando en el discurso de nuestros informantes se constata que domina:

- La marca en el voto tradicional a la marca de siempre, en el caso de los nuevos partidos, en la vuelta a lo de siempre (PSOE y PP) tras la frustración con las expectativas de cambio.

- El liderazgo cuando aparece un mirlo blanco, cuando se asocia a la expectativa de cambio o a la marca habitual, cuando domina la incertidumbre de qué votar.

- La expectativa de cambio en las etapas de crisis política como la actual, en la transferencia del voto a los nuevos partidos, en la proyección del voto nacionalista en Nueva Canarias.

- La conformidad con la opción tomada en las épocas en que se mantiene la percepción de mejora, en las etapas de permanencia de un líder carismático.

En los tres municipios estudiados se confirma que el/la candidata(a)/persona ha tenido un peso específico en la configuración del voto en los comicios locales realizados en dichos municipios en las últimas décadas. 
Era el propio discurso de nuestros/as informantes la que nos marcaba con claridad una diferencia singular en la configuración del voto en las elecciones locales señalando a la condición personal del candidato/a como principal diferencia.

"En lo local se vota a las personas" es una opinión que alcanza un respaldo mayoritario en el conjunto de los grupos.

El avance en el intercambio dinámico de los grupos sobre la evolución percibida/ experimentada en cada municipio nos ha permitido dimensionar el alcance de esa opinión:

- El liderazgo/candidato/persona aparece como una variable interviniente en la mayoría de los casos (elecciones municipales) estudiados.

- El principal motivo aducido para argumentar esta percepción social es la cercanía.

- De ahí que el peso atribuido al liderazgo en la configuración del voto local se vaya incrementando según se reduce el tamaño de las poblaciones.

La profundización en el discurso de nuestros/as informantes nos permite relativizar la persuasión de partida: el liderazgo/candidato/persona comparte la explicación (co causalidad) de lo experimentado con otros tres elementos (la marca, la expectativa de cambio y la conformidad con la opción tomada.) y en varios de casos referenciados no aparece como factor dominante.

El análisis de las lógicas argumentales en las que se sustentan las anteriores percepciones sociales nos permite graduar la intervención atribuida al liderazgo/candidato/persona en una escala nominal:

- Una intervención minoritaria. Así se comprueba en las últimas elecciones municipales en Las Palmas de Gran Canaria, el caso donde el liderazgo presenta un menor peso. La falta de notoriedad de los candidatos a la alcaldía en esa circunstancia hizo que en la configuración del resultado electoral dominara el voto a la marca, tanto en el mantenimiento del suelo electoral de los partidos estatales "de siempre" como en el tirón electoral de alguno de los nuevos partidos. Aun así, comprobábamos que las figuras de Antonio Morales y de José Miguel Bravo de Laguna habían tenido una incidencia significativa en el incremento del voto de dos formaciones minoritarias, Nueva Canarias y Unidos por Gran Canaria al captar para estas marcas parte de los electores de CC y del PP.

- Cabe destacar que la nueva configuración actual del espectro electoral, más allá del bipartidismo, resalta la importancia que adquieren estas formaciones políticas minoritarias para la formación de los equipos de gobierno municipales.

- Una intervención decisiva en aquellos casos en los que la atribución del liderazgo político local a un candidato/a sirve para conseguir mayorías absolutas al captar votantes de otras posiciones ideológicas que votan a otros partidos en comicios no municipales. Así lo comprobábamos en la consolidación de José Manuel Soria como alcalde de Las Palmas en las sucesivas legislaturas en las que se mantuvo como regidor de la ciudad, y en la mayoría absoluta con que fue elegido Jerónimo Saavedra.

- Una intervención determinante cuando el atractivo del líder supera al de la marca y: 
- consigue mantener la adhesión a una marca propia (el PIL de Dimas Martín en Teguise)

- sustenta la recuperación de mayorías de la marca (Oswaldo Betancort en Teguise)

- incluso posibilita al candidato el cambio de marca manteniendo mayorías electorales y/o alcaldías (Juan Pedro Hernández en Teguise, Gladys Acuña en Yaiza y Pedro Martín en Santa María de Guía).

\subsection{Las cualidades que configuran el liderazgo político local}

El análisis de las cualidades que configuran el liderazgo político local exigen analizar la condición singular del liderazgo político local, las condiciones de personalidad señaladas por las teorías reseñadas y la secuencia de las dimensiones de personalidad en el liderazgo político local.

\subsubsection{La condición singular del liderazgo político local}

La primera constatación a destacar es que las teorías sobre las dimensiones de personalidad, a las que hemos hecho referencia anteriormente, es su carácter multidimensional como apunta Rico (2009). La segunda es que no consideran la diferencia que singulariza los comicios locales con respecto a los procesos electorales. De ahí que no contemplen el elemento básico que marca esta diferencia: la cercanía del político candidato a los ciudadanos.

La falta de cercanía a la gente, al ciudadano, a los barrios de la ciudad, aparece como el principal motivo de que el liderazgo político apenas haya tenido una intervención minoritaria en el resultado de la configuración del voto en las últimas elecciones al Ayuntamiento de La Palmas de Gran Canaria. Por el contrario, todavía se mantiene en la memoria de nuestros/a informantes el recuerdo de Nardi Barrios, quien fue candidata a la alcaldía al municipio en pasadas convocatorias electorales, cuyo reconocimiento social se mantiene asociado a su empeño por mantenerse cercana a la gente, a los barrios y a los problemas que los afectan.

"Nardi iba a las asociaciones de vecinos. Los visitaba habitualmente para conocer su situación y recoger sus demandas".

La cercanía, el saber llegar a la gente haciéndose sentir cercano a ella, aparece como la principal cualidad atribuida al anterior alcalde de Santa Lucía de Tirajana, Silverio Matos, a quien se sigue reconociendo como el principal líder social/local en el municipio. La falta de esta cualidad se señala como la principal pérdida resentida en el recambio generacional producido en la alcaldía y como la principal carencia de la nueva alcaldesa y nueva candidata por Nueva Canarias.

"El anterior alcalde era muy cercano, te saludaba, hablabas con él como con cualquier persona; la actual no llega a tanto, no tiene la misma cercanía" (Santa Lucía de Tirajana).

"Le veo muy cercano y me da sensación de buena gente agradable y atento, me da confianza" (Teguise). 
El ser percibido como una persona cercana a la gente aparece como la persuasión básica sobre la que se ha sustentado la imagen de Oswaldo Betancort como el mejor alcalde de Teguise en las últimas décadas y su reconocimiento como el nuevo líder político local, e incluso insular.

El caso de las últimas elecciones municipales al Ayuntamiento de Las Palmas de Gran Canaria nos proporciona otra importante lección a considerar: la carencia de la notoriedad suficiente como para alcanzar un reconocimiento propio, imposibilita la valoración del liderazgo y de su incidencia en los procesos electorales, pues la falta de notoriedad/reconocimiento se traduce en la imposibilidad de proyectar atributos personales y atribuciones políticas a los candidatos/as, así como de poder establecer alguna valoración de su trayectoria política.

"Es que el nuevo alcalde no se ni quién es" (en referencia a Augusto Hidalgo, actual alcalde de Las Palmas de Gran Canaria).

Así pues, la notoriedad de los candidatos/as y la cercanía a los ciudadanos aparecen como las condiciones de personalidad prioritarias para el reconocimiento del liderazgo político a nivel local.

\subsubsection{Las condiciones de personalidad señaladas por las teorías reseñadas}

La sistematización de los elementos recogidos en la tabla 1 en la que se resumen las quince teorías reseñadas anteriormente, ofrece la siguiente jerarquización:

- La competencia y la integridad / honradez son las dos variables más señaladas por las diferentes teorías del liderazgo.

- El liderazgo del candidato y su carisma logra también una elevada relevancia al indagar las dimensiones de la personalización.

- La empatía es citada por algunos autores en estudios de elecciones de primer orden, lo mismo que las características sociodemográficas y personales. Como veremos, aunque presentes no son nucleares en la personalización del voto en elecciones municipales.

El análisis realizado nos permite concluir que la competencia es una dimensión de personalidad que aparece destacada de forma transversal en la valoración del liderazgo político local. Y lo dicho anteriormente nos permite avanzar en el dimensionamiento de esta variable en tres sentidos:

- Como formación/preparación/capacitación. Este "valor"/significado aparece atribuido a los nuevos líderes políticos, que personalizan la renovación generacional a nivel local y/o insular. Así lo constatábamos en el caso de la nueva alcaldesa de Santa Lucía de Tirajana, del nuevo alcalde de Teguise y del conjunto de los nuevos políticos que conforman la renovación generacional en Lanzarote.

- "Ella (alcaldesa de Santa Lucía) es técnica. Es muy trabajadora, diría que más gestora que cercana".

- "Oswaldo creo que es abogado o algo así" (Teguise).

- Como la trayectoria política vivida por el candidato/a. Así lo constatábamos en el caso de Jerónimo Saavedra, al ser elegido como alcalde de Las Palmas 
de Gran Canaria. De él se dice que ha sido de todo en la política canaria, e incluso en el Gobierno de Madrid.

- "Es que Jerónimo (Saavedra) lo ha sido todo, incluso Ministro".

- Como la capacidad de gestión probada por los resultados conseguidos y las mejoras percibidas en los municipios. Así lo hemos podido constatar en los casos de José Manuel Soria como alcalde de Las Palmas, de Dunia González como alcaldesa de Santa Lucía de Tirajana y de Oswaldo Betancort como alcalde de Teguise.

La integridad del candidato/a aparece también referenciada en algunos de los casos estudiados. Y lo hace como una dimensión que lleva a retraer parte del voto acumulado por la marca o por el líder/candidato debido a tres motivos principales:

- El transfuguismo. Hemos visto cómo una parte de los electores de CC se manifiestan dispuestos a replantearse su voto si su candidatura apareciera encabezada por una figura política que carga con el san Benito de chaquetero.

- La corrupción. Hemos podido constatar la progresiva pérdida de votos del PIL, la marca propia de Dimas Martín según el líder se iba viendo afectado por sucesivas sentencias judiciales.

- La falta de transparencia. Algunos de nuestras/os informantes señalan en este sentido al valorar la respuesta dada por el alcalde de Teguise ante la demanda de rendir cuentas por parte de un partido de la oposición.

Conviene destacar que esta dimensión opera más bien "por defecto" cuando determinadas noticias alcanzan una significativa incidencia en la opinión pública local. Mientras no sea así, tiende a no estar presente en la valoración del liderazgo político, como se refleja claramente en el discurso de nuestros informantes: "De momento no hay por qué dudar...Hasta ahora no se ha sabido nada en ese sentido."

Ya se señaló, al tratar estos casos, que la dimensión de integridad aparece con un sentido ambivalente: aunque llegar a devaluar la imagen del líder y la adhesión del voto de una parte de sus electores, otra parte de su electorado mantiene la defensa del mismo argumentando en base a sus aspectos positivos. El caso de Dimas Martín resulta arquetípico en este sentido.

El mismo Dimas Martín aparece, entre los casos estudiados, como el líder local al que nuestros/as informantes atribuyen un mayor carisma político. Esta atribución se sustenta principalmente en el hecho de reconocer en él una enorme capacidad de convicción, tanto a nivel colectivo como personal: La gente iba a oírle y acababa convencida de lo que les decía... Incluso algunos que iban al ayuntamiento muy cabreados porque se sentían perjudicados por él salían de allí tan contentos con las explicaciones que les había dado y terminaban tomándose un café juntos.

La empatía no aparece referida directamente, aunque tal vez podría contemplarse como una dimensión de personalidad incluida dentro de la cercanía.

En cuanto a las características socio demográficas se destaca el sexo, como una dimensión de personalidad que aporta un valor especial a la elección de Dunia González como alcaldesa de Santa Lucía de Tirajana, y la edad, especialmente destacada en la llegada de Oswaldo Betancort a la alcaldía de Teguise. 


\subsubsection{La secuencia de las dimensiones de personalidad en el liderazgo político local}

De todos los casos estudiados es el de Oswaldo Betancort el que destaca especialmente en cuanto a la percepción social de un líder político local. El discurso de nuestros/as informantes nos posibilita reconstruir la evolución percibida desde que es elegido como candidato de CC a la alcaldía de Teguise hasta su consolidación como alcalde en su segunda legislatura. El análisis de dicha evolución nos permite avanzar que en la construcción social de la imagen de líder político local intervienen tres tipos de elementos/variables/cualidades que hacen referencia a:

- Cualidades de tipo personal tales como la edad, el trato, el talante, la actitud de respuesta a los demás.

- Su corrección política; a su cercanía a los ciudadanos, a los lugares donde residen y a los problemas que les afectan; a su accesibilidad y asequibilidad como gobernante local.

- Los resultados conseguidos y a la percepción de mejora en la ciudad.

La distinta incidencia con que aparecen estos tipos de variables a través del tiempo contemplado nos permite establecer un dimensionamiento del peso de cada uno de estos factores en la trayectoria analizada estableciendo una secuencia en tres momentos/tramos. Aun cuando aparecen referencias explícitas a los tres tipos de variables en cada uno de los tramos, el análisis realizado nos lleva a constatar que:

- En el primero de ellos dominan las referencias a las cualidades de tipo personal: alguien joven con nuevas ideas y proyección de futuro; con un trato, afable, amable, dulce, correcto y elegante; a quien se siente cercano. La imagen de Oswaldo Betancort en este primer momento está dominada por los significados de buen chico, buena gente, buen talante...buena persona y cercana.

- En el segundo momento dominan las referencias a su corrección política; a la confianza que transmite como político y a la accesibilidad como gobernante local: es trabajador, te escucha en cualquier parte, puedes acceder a él directamente, te atiende personalmente, está ahí cuando lo necesitas. La imagen de Oswaldo Betancort en este segundo momento está dominada por un significado prioritario: está para el pueblo.

- En el tercer momento pasan a tomar una especial relevancia las referencias a los resultados de la gestión realizada como alcalde y las mejoras percibidas en el municipio y en el equipo de gobierno: se ha contrarrestado la sensación de abandono de algunas zonas del municipio (Costa Teguise, Caleta de Famara), se han experimentado sensibles mejoras en las infraestructuras aun cuando no hayan alcanzado todavía a algunos atrases y pueblitos del municipio, se destaca la acción del equipo de gobierno en su conjunto y la eficiencia de algunos de sus miembros en la gestión de los asuntos municipales. La imagen de Oswaldo Betancort en este tercer momento se ve confirmada como el mejor alcalde de la ciudad en mucho tiempo.

\footnotetext{
"Una buena persona y cercana a la gente".

"Un gobernante que está a disposición del pueblo".

"El mejor alcalde de la ciudad en mucho tiempo".
} 


\section{Conclusiones}

El estudio del comportamiento electoral a nivel local es un campo escasamente investigado hasta hace unos pocos años por la ciencia política. Es así en buena parte de las democracias con más historia y también lo es en el caso de España. La percepción del nivel local como un entorno más administrativo que político y con recursos más limitados que las comunidades autónomas y el propio gobierno central, ha relegado a las elecciones locales a un segundo plano en las investigaciones del comportamiento electoral. A todo lo anterior debe añadirse la dificultad intrínseca de investigar con rigurosidad (y, sobre todo, capacidad de generalización) los más de 8.000 municipios que existen actualmente en nuestro territorio, el reto que en muchas ocasiones supone disponer de datos agregados y plenamente comparables y la maraña de partidos y coaliciones que se presentan únicamente en un municipio, singularizando el territorio y complicando aún más el análisis de las elecciones municipales.

Sin embargo, la relevancia de los ayuntamientos ha crecido en las últimas décadas (en parte por eventos negativos, como la corrupción política) gracias a la relevancia de los presupuestos - particularmente en las capitales de provincia- y en parte por el creciente protagonismo de sus alcaldes, que excede del ámbito meramente local. Sirva como ejemplo las actuales alcaldesas de Barcelona o Madrid; e incluso en los casos estudiados la figura de Oswaldo Betancort -alcalde de Teguise- que alcanza proyección insular.

La investigación realizada ha podido constatar que en el discurso social sobre el proceso decisional del voto en los comicios municipales el binomio marca / partido - líder / candidato que afirman las teorías sobre elecciones de primer orden están también presentes en el ámbito local. Sin embargo, en el discurso de los votantes aparecen otras dos variables que explican cualitativamente su comportamiento: la expectativa de cambio y la conformidad con la opción elegida en el pasado.

- La expectativa de cambio actúa como una variable operante, que adquiere protagonismo frente al resto cuando se desea dar paso a nuevas alternativas ("por probar algo diferente"); para compensar la decepción por una elección fallida; o bien para salir de una situación de impasse previo. Una lógica argumental que se ha podido experimentar con la irrupción de Podemos y sus marcas satélites en 2015 en miles de ayuntamientos; y su previsible ajuste a tenor de los estudios de intención de voto más recientes.

- La conformidad con la opción elegida, sin embargo, es una variable secundaria concomitante, que actúa reforzando la elección de la marca / partido o candidato / líder cuando la mejora en la percepción del municipio es positiva.

En cuanto a la variable candidato / liderazgo personal, los resultados del análisis de los tres casos estudiados vienen a conferir a esta variable tres grados de intensidad:

- Es una variable interviniente en todos los casos estudiados. El candidato en las elecciones municipales resulta nuclear en el proceso decisional del voto.

- El candidato resulta decisivo en aquellos escenarios locales en los que es útil para alcanzar mayorías absolutas, captando votantes que en elecciones estatales (e incluso autonómicos y/o insulares) optan por otras siglas. 
- Y es determinante cuando el atractivo del líder local supera al de la marca que representa, sustentando a ésta, e incluso validando el cambio a otras siglas políticas. Esto último se ha podido verificar en los últimos comicios municipales en Yaiza o en Santa María de Gruía.

La multidimensionalidad del liderazgo personal que ya anunciaban las diferentes teorías sobre la personalización, se confirma en las elecciones municipales. Tanto la competencia del candidato, como su integridad y honradez quedan reflejadas en el discurso de los electores cuando se refieren a elecciones municipales. Pero la investigación cualitativa realizada añade dos nuevas variables a considerar:

Por un lado, la notoriedad, que ya apuntaba Riera (2017) al referirse a la relevancia del tamaño del municipio, aunque en el análisis del discurso de nuestros informantes la notoriedad se jerarquiza como una variable imprescindible para que el liderazgo personal actúe, con independencia del tamaño del municipio. Expresado en términos de marketing, no puedo comprar un producto del que ni siquiera tengo consciencia de su existencia. No puedo, en definitiva, votar por un candidato basándome en su liderazgo personal cuando no tengo el más mínimo conocimiento de su persona (sí puedo votar a la marca, pero no al candidato).

Por otro, la cercanía del candidato con el electorado, que opera en tres niveles distintos del liderazgo personal:

- En lo personal: Se circunscribe al aspecto más inmediato, la imagen previa ("Es joven" "es buena gente") a la que se puede acompañar de algún conocimiento o experiencia básica con el líder (haber ido a la misma escuela, tener amigos en común, haber charlado en alguna ocasión, ...).

- En lo político: Es el siguiente tramo de la cercanía. Se asocia con la atención que presta el líder a las cuestiones ajenas personales ("Te atiende" "te escucha" "se preocupa" "intenta resolver tu problema"). No alcanza el estatus de gestión sino más bien de capacidad de resolver asuntos o problemas puntuales que requieren de una respuesta no dilatada en el tiempo.

- En la mejora percibida: Que aparece en el discurso sobre el líder político cuando su actuación influye positivamente en las mejoras percibidas en el municipio y además se responsabiliza al líder de dicho logro.

La investigado ha ahondado en la personalización del voto en las elecciones municipales, identificando las variables que compiten en el procedo decisional del voto local más allá del candidato; y ha profundizado en la muldimensionalidad de estas variables desde la óptica de los comicios locales. Una tarea que resulta de especial interés cuando acabamos de terminar elecciones municipales en 2019, en las que se podrán contrastar de manera empírica los descubrimientos que aquí se han aproximado. Tarea que se ha llevado a cabo mediante la aplicación de metodologías cuantitativas que posibiliten la verificación de las hipótesis que aquí se han esbozado.

\section{Bibliografía}

Barberá, P. (2010). "Voting for parties or for candidates? The trade-off between party and personal representation in Spanish regional and local elections", en Revista Española de Investigaciones Sociológicas, 132: 35-63 
Bartie, J. e I. Crewe (2002). "The impact of party leaders in Britain: Strong assumptions. Weak evidence”, en A. King. (eds.). Leader's personalities and the outcomes of democracy elections. Oxford: Oxford University Press.

Bean, C. (1993). "The electoral influence of party leader images in Australia and New Zealand", en Comparative Political Studies, 26: 111-132

Capo, J. (1991). "elecciones municipales, pero no locales”, en Revista Española de Investigaciones Sociológicas, 56: 143-164

Clarke, H. (2000). "Major's lesser effects. Prime ministerial approval and governing party support in Britain since 1979", en Electoral Studies, 19: 255-253

Chetty, S. (1996) "The case study method for research in small and medium sized firms", en International Small Business Journal, 15 (1): 73-85.

Costa Lobo, M. (2014). "Party and electoral leadership". en M. Rhodes (eds.). The Oxford handbook of political leadership. Oxford: Oxford University Press

Costa Lobo, M. y J. Curtis (2014). Personality politics? The role of leader evaluations in democratic elections. Oxford: Oxford University Press

Delgado, I. (1997). El comportamiento electoral municipal español 1979-1995. Madrid: Centro de Investigaciones Sociológicas

Delgado, I. (1997). “elecciones municipales en España. Dimensiones analíticas y aspectos distintivos de ocho procesos electorales" (1997-2007)", en Politicas y Sociedad, 47: 1336

Freire, A. (2005). "Eleicoes segunda ordem e ciclos eleitorais no Portugal democrático, 1975-2004", en Analise Social, 177: 815-846

Graetz, B y McAllister, E. (1987). "Party leaders and electoral outcomes in Britain, 19741983”, en Comparative Political Studies, 19: 484-507

Hazan, R. (1996). "Presidential parliamentarism: Direct popular election of the Prime Minister”, en Electoral Studies, 15: 21-38

King, A. (2002). “Conclusions and implications”, en A. King (eds). Leader's personalities and the aoutcomes of democratic elections. Oxford: Oxford University Press

Márquez, G. (2007). Política y gobierno local. La formación de gobierno en las entidades locales en España. Madrid: Centro de Estudios Políticos y Constitucionales

Marschall, M, P. Shan y A. Ruhil (2011). "The study of local elections", en Political Science \& Politics, 44: 97-100

Martínez, P. C. (2006). "El método del estudio de caso. Estrategia metodológica de la investigación científica", en Pensamiento y Gestión, 20: 165-193

Martínez Fuentes, G. (2008). "Local political leadership in Spain”, en Local Government Studies, 34: 267-278

Martínez Fuentes, G. y C. Ortega (2010). “The political leadership. Factor in the Spanish local elections", en Lex Localis. Journal of Local Self- Government, 8: 147-160

Mughan, A. (1993). "Party leaders and presidentialism in the 1992 british election and parties yearbook", en D. Denver, P. Norris y C. Rallings (eds). British elections and parties yearbook. Londres: Harvester Wheatsheaf, pp 193-204.

Rico, G. (2007). “¡No nos falles! Los candidatos y su peso electoral”, en J.R. Montero, I. Lago y M. Torcal (eds). Elecciones generales 2004. Madrid: Centro de Investigaciones Sociológica.

Rico, G. (2009) Líderes políticos, opinión pública y comportamiento electoral en España. Madrid. Centro de Investigaciones Sociológicas

Riera, P., Gómez, R., Mayoral, J.A., Barberá, P y Montero, J. R. (2017). “elecciones municipales en España. La personalización del voto”, en Revista Internacional de Sociología, 75: 1-18. 
Sarabia, F. J. (1999). Metodología para la investigación en Marketing y dirección de empresas. Madrid: Pirámide

Vanclocha, F.J: (1997) “Identidad de los gobiernos locales y reforma electoral”, en C. R. Alba y F.J. Vanaclocha (eds.). El sistema político local: un nuevo escenario de gobierno. Madrid: Universidad Carlos III

Yin, R.K. (1993). Applications of case study research. Applied social research methods series. Sage: California. 